\title{
Control of leaf vascular patterning by polar auxin transport
}

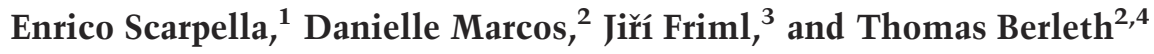 \\ ${ }^{1}$ Department of Biological Sciences, University of Alberta, Edmonton, Alberta T6G 2E9, Canada; ${ }^{2}$ Department of Botany, \\ University of Toronto, Toronto, Ontario M5S 3B2, Canada; ${ }^{3}$ Center for Plant Molecular Biology (ZMBP), Tübingen \\ University, D-72076 Tübingen, Germany
}

\begin{abstract}
The formation of the leaf vascular pattern has fascinated biologists for centuries. In the early leaf primordium, complex networks of procambial cells emerge from homogeneous subepidermal tissue. The molecular nature of the underlying positional information is unknown, but various lines of evidence implicate gradually restricted transport routes of the plant hormone auxin in defining sites of procambium formation. Here we show that a crucial member of the AtPIN family of auxin-efflux-associated proteins, AtPIN1, is expressed prior to pre-procambial and procambial cell fate markers in domains that become restricted toward sites of procambium formation. Subcellular AtPIN1 polarity indicates that auxin is directed to distinct "convergence points" in the epidermis, from where it defines the positions of major veins. Integrated polarities in all emerging veins indicate auxin drainage toward pre-existing veins, but veins display divergent polarities as they become connected at both ends. Auxin application and transport inhibition reveal that convergence point positioning and AtPIN1 expression domain dynamics are self-organizing, auxin-transport-dependent processes. We derive a model for self-regulated, reiterative patterning of all vein orders and postulate at its onset a common epidermal auxin-focusing mechanism for major-vein positioning and phyllotactic patterning.
\end{abstract}

[Keywords: Arabidopsis; AtPIN genes; polar auxin transport; procambium; leaf development; vascular patterning]

Supplemental material is available at http://www.genesdev.org.

Received December 19, 2005; revised version accepted February 16, 2006.

The vascular system of plants is composed of a continuous network of vascular bundles, primarily composed of phloem and xylem tissues that translocate dissolved photoassimilates and minerals, respectively (Esau 1965). In leaves, all vascular cells differentiate from a vascular meristematic tissue, the procambium. Procambial cells become apparent as narrow, cytoplasm-dense cells emerging from the subepidermal tissue of the leaf primordium, termed ground meristem (Foster 1952; Esau 1965). Procambial cells are characteristically arranged in continuous strands and acquire their narrow shape through coordinated, oriented divisions, parallel to the axis of the emerging strand.

Few natural phenomena have attracted more widespread attention, including reflections by mathematicians, philosophers, and theoretical biologists, than the so-called venation patterns of leaves. From a developmental point of view, the patterning process is particularly intriguing, because it seems to be organized de novo in each leaf primordium. Moreover, a considerable de-

${ }^{4}$ Corresponding author.

E-MAIL berleth@botany.utoronto.ca; FAX (416) 978-5878.

Article and publication are at http://www.genesdev.org/cgi/doi/10.1101/ gad.1402406. gree of variability of vein patterns in most species argues against their rigid specification and suggests instead highly flexible self-organizing patterning mechanisms. These mechanisms must reconcile the plasticity of vein patterns with a stringent continuity requirement for vascular function.

Most vascular patterning models fall in two categories distinguished by a basic alternative: In one, flow of a signal through incipient vascular strands is critical for the patterning process (Sachs 1981, 1991); in the other, vascular strand patterns are specified through cellular interactions in the surrounding tissue, for example, through autocatalytic, local-activation-long-range-inhibition mechanisms (Meinhardt 1982; H. Meinhardt and A. Gierer, http://www.eb.tuebingen.mpg.de/dept4/ meinhardt/theory.html). A crucial formal distinction between the two self-organizing models is that signal flow involves continuous signaling through the specified cells and thereby requires that all vascular strands are necessarily connected at least transiently at an early stage, while local-activation-long-range-inhibition mechanisms could generate entirely disconnected vascular strands.

An instrumental role of auxin transport and auxin transport-mediated auxin distribution for tissue pattern- 
ing in young plant organs has gained much support from the results of auxin transport-inhibition studies and auxin application studies in combination with new cellular markers (Leyser 2005). Control of auxin transport seems to be the single most critical parameter for the establishment and dynamic maintenance of auxin distribution patterns in, for example, embryos and shoot and root meristems. Controlled auxin transport, in turn, seems to be accurately visualized through the expression pattern and subcellular localization of auxin-efflux-associated proteins of the AtPIN (PIN in the following) family.

Despite considerable work in tracing pre-procambial development and in identifying mutants with abnormal venation (Turner and Sieburth 2002), the patterns of auxin transport routes and their relationship to procambium have remained unknown. Here we monitor these patterns through stages critical for the formation of various defined vein classes in rosette leaves of Arabidopsis. Our data reveal strongly coordinated acquisition of subcellular polarity in all cells contributing to procambium formation for all vein classes and a directing influence of auxin transport in the epidermis on vein patterning. Despite the vast diversity of vein shapes and connections in the vascular network, these data suggest that two types of vein ontogeny are sufficient to explain the complexity of Arabidopsis vein patterning.

\section{Results}

For clarity, we summarize our schemes for reporting positions within leaf primordia and for vein orders in Figure 7A, below. The representative character of each reported feature is indicated as a quotient (samples showing the feature/all samples) in the lower left corner of Figures 1-6, with the criterion features listed in Supplementary Table S1.

Expression and subcellular polarities of PIN proteins have been in good agreement with independently assessed auxin distributions and flow directions (for review, see Leyser 2005). Furthermore, auxin transport is reduced in pin1 mutants, and a spectrum of phenotypic features, including altered vascular patterns, can be mimicked by chemical inhibition of auxin transport (Okada et al. 1991; Mattsson et al. 1999; Koizumi et al. 2005). Finally, PIN protein localizations have recently been shown to be critical for defining the direction of auxin flow in Arabidopsis roots (Wisniewska et al. 2006). In order to disentangle auxin transport routes preceding procambium specification in the leaf, we first determined through transcript profiling that, of this family, only PIN1, PIN3, PIN4, and PIN7 are expressed in the first pair of leaf primordia (first leaf in the following) at 5 $\mathrm{d}$ after germination (DAG) (data not shown). Of these four genes, only PIN1 is expressed early in ground meristem cells as judged from the expression patterns of a functional translational reporter (Fig. 1). In contrast, PIN3, PIN4, and PIN7 are either not expressed in ground meristem cells or are expressed clearly after procambium has been formed (Supplementary Fig. S1). We conclude
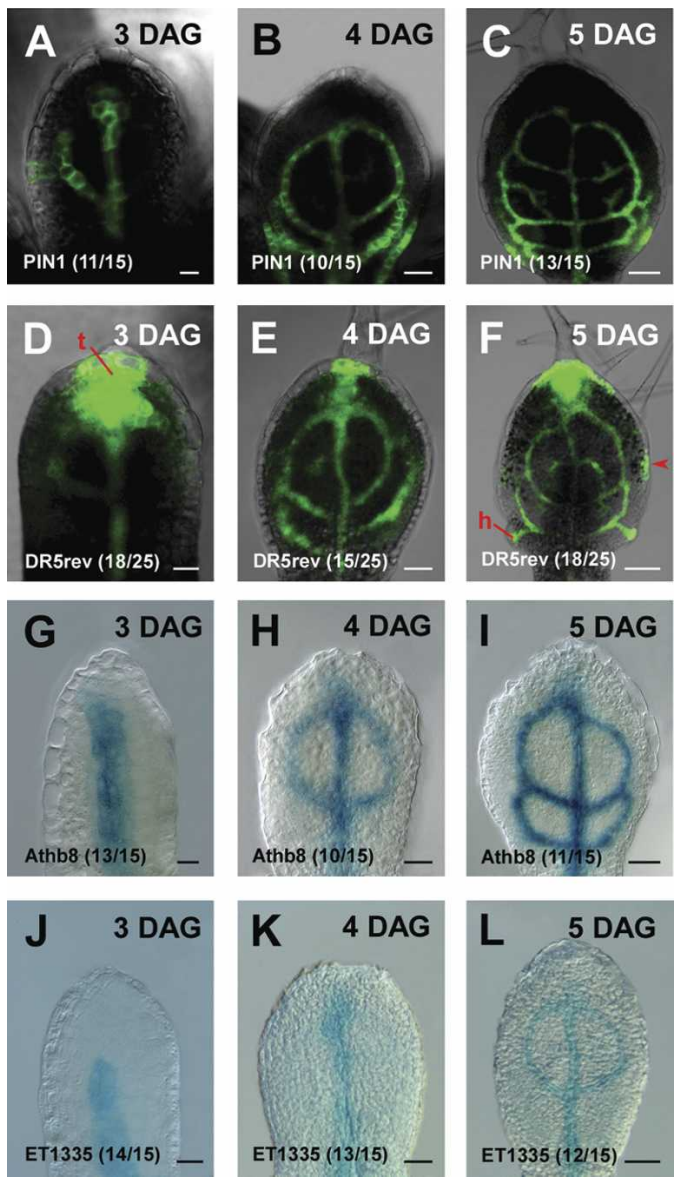

Figure 1. Consecutive appearance of gene expression markers during leaf procambium specification. (Upper right) Primordium age in days after germination (DAG). (Lower left) Marker identity. (Parentheses) Fraction of primordia showing the displayed features (Supplementary Table S1). All markers are expressed in all vein classes. Note that expression of PIN1: GFP and DR5rev::GFP precede Athb8::GUS expression by nearly a day. (ET1335) Procambium marker. Note epidermal DR5rev::GFP expression at the primordium tip $(\mathrm{t})$, hydathodes (h), and in discrete areas of the lateral epidermis (red arrowhead, F). Arabidopsis first-leaf primordia, primordium orientation: tip toward top. $(A-F)$ GFP fluorescence and transmitted light. $(G-L)$ DIC microscopy. Bars: $A, D, G, J, 10 \mu \mathrm{m} ; B, E, H, K, 20 \mu \mathrm{m} ; C, F, I, L$, $50 \mu \mathrm{m}$.

that for leaf vein patterning, PIN1 is presently the only informative marker within the PIN gene family. The use of a functional PIN1:GFP translational fusion with expression profiles indistinguishable from those of immunolocalized PIN1 protein (Benkova et al. 2003) allowed for reproducibility assessment of all reported features in hundreds of optimally oriented whole-mount specimens (Supplementary Table S1).

We next asked whether PIN1 expression reliably precedes procambium formation. A sharp criterion to answer this question is its expression relative to the pre-procambial marker Athb8::GUS. This marker has been shown to precede the formation of procambial cells and the simultaneous expression of the procambial 
marker ET1335 in all vein classes (Fig. 1; Kang and Dengler 2004; Scarpella et al. 2004). As is shown in further detail in the following sections, PIN1 is expressed in, and occasionally around, all incipient veins and consistently precedes the expression of the Athb8::GUS marker by nearly a day (Fig. 1A-C,G-I). We conclude that PIN1: GFP expression domains (PEDs) coincide with the probable sites and stages at which pre-procambial cells are being selected from the ground meristem and therefore monitored PED dynamics and PIN1:GFP subcellular polarity in normal and experimentally manipulated leaf primordia.

\section{Initiation of lower-order veins}

In epidermal cells of the shoot meristem, PIN1 protein becomes localized toward the initiation sites of new leaf primordia. It has been postulated that at these sites, auxin converges and is then transported (internalized) from the epidermis to subepidermal tissues (Benkova et al. 2003; Reinhardt et al. 2003). We asked whether auxin internalization leads to preferred routes of auxin transport in the ground meristem of leaf primordia (rosette leaves $1-8$ ). In very early leaf primordia, convergent (opposed) polarity of PIN1 protein toward a central point in the epidermis ("convergence point," highlighted by signal quantification in Fig. 2D,E) is associated with a central, subepidermal PED, in which PIN1 polarity is basally oriented (Fig. 2E). In the following, we use "basal" to describe PIN1 subcellular polarity oriented toward pre-existing vasculature, regardless of how a vein is positioned within a leaf, and "centrobasal" when basal polarity is skewed toward the center of a PED. As the primordium quickly acquires an elongated, cylindrical shape, PIN1:GFP is expressed in basal polarity along the entire incipient midvein (Fig. 2G,H). Especially in the most distal part, the PED may be wider with centrobasal subcellular polarities (Fig. 2G). Epidermal PIN1 expression is remarkably homogeneous at this stage of invariably apical polarity (Fig. 2F).

The appearance of veins connected to pre-existing vasculature at both ends ("connected veins") has puzzled observers and has spurred discussions on the applicability of signal-flow models to this type of veins (Nelson and Dengler 1997). We used the continuous loops of second-order veins, which are formed with high regularity early in leaf development, as an opportunity to study the ontogeny of connected veins. PEDs of second-order veins emerge in association with epidermal convergence points (Fig. 2I-N), analogous to the midvein (first order) PED. These convergence points are located at the leaf margin of 2.5- and 3-DAG primordia (for the most distal pair of loops) approximately half-way from the tip (Figs. 2H,K-M, 7C [below]). Interestingly, convergence points are invariably positioned at the leaf margin and are not observed in other parts of the leaf epidermis, suggesting an exclusive function of the leaf margin in directing ground meristem patterning (see Discussion).

Beyond the formation of convergence points, there is more similarity in the ontogeny of first- and second-order veins. At 3 DAG, second-order PEDs appear to connect each convergence point with the midvein PED. These early second-order PEDs represent only the lower (proximal) part of a loop (lower-loop domain, LLD). Each LLD comprises a transient wide section near the convergence point and a persistent narrow proximal section (Figs. 2K-N, 7C [below]; Supplementary Movie S1), the latter giving rise to second-order vein procambium. The polarity of subcellular PIN1 localization is basal or centrobasal in the distal section and basal in the proximal section (Fig. 2K-N). By 3.5 DAG, the initially wide distal section eventually disappears (Fig. 2O,P). In conclusion, second-order vein loops are of composite origin. The ontogeny of the LLD is reminiscent to that of the midvein PED including the presence of an ephemeral, often wider distal and a narrow proximal section.

The formation of the complementary "upper-loop domain" (ULD) is a separate event (Fig. 7C, below; Supplementary Movie S1). The ULD is typically initiated after the LLD is fully connected to the midvein PED and is then gradually extended from the LLD toward the distal midvein (Figs. 2N,O, 7C [below]; Supplementary Movie S1). These features might be important, because the polarity in the ULD is directed toward the LLD through which it is connected to the midvein PED (Fig. 2N,O). Therefore, its polarity is basal with reference to the LLD, which is a pre-existing PED at this stage. As the ULD is extended toward the leaf tip, it finally becomes connected to the midvein PED at its distal end and becomes a "connected" PED (Fig. 2P). Most remarkably, those connected ULDs are invariably comprised of two segments of opposite polarity, bridged by a single bipolar cell (Fig. 2P-U). Each segment's polarity is invariantly basal and is smoothly coordinated with the polarity of the adjoining pre-existing PED. There is never more than a single polarity switch and a single bipolar cell within a ULD.

As is shown below, this bipolar organization is a general feature of all "connected" PEDs in all Arabidopsis vein orders. Therefore, ontogenetically, the formation of the conspicuous second-order vein loops is composite, with each part conforming to a more general principle: The formation of an epidermally initiated LLD is similar to that of the midvein, while the ontogeny of the ULD resembles that of connected PEDs as they are formed in higher-order veins throughout leaf development (below).

The subsequent formation of more proximal vein loops $(12,13)$ reiterates the features observed in the first loop. Convergence points are again restricted to the margin of the leaf lamina and are connected to the basal part of the midvein through characteristic distal and proximal sections (Supplementary Fig. S2). One feature distinguishing those loops from the first pair of loops is the fact that ULDs connect distally to the next distal secondorder loop rather than to the midvein (Supplementary Fig. S2). A second feature is of a quantitative nature. Especially visible in third loop LLDs and reiterated in second-order veins of larger leaves formed later in the 

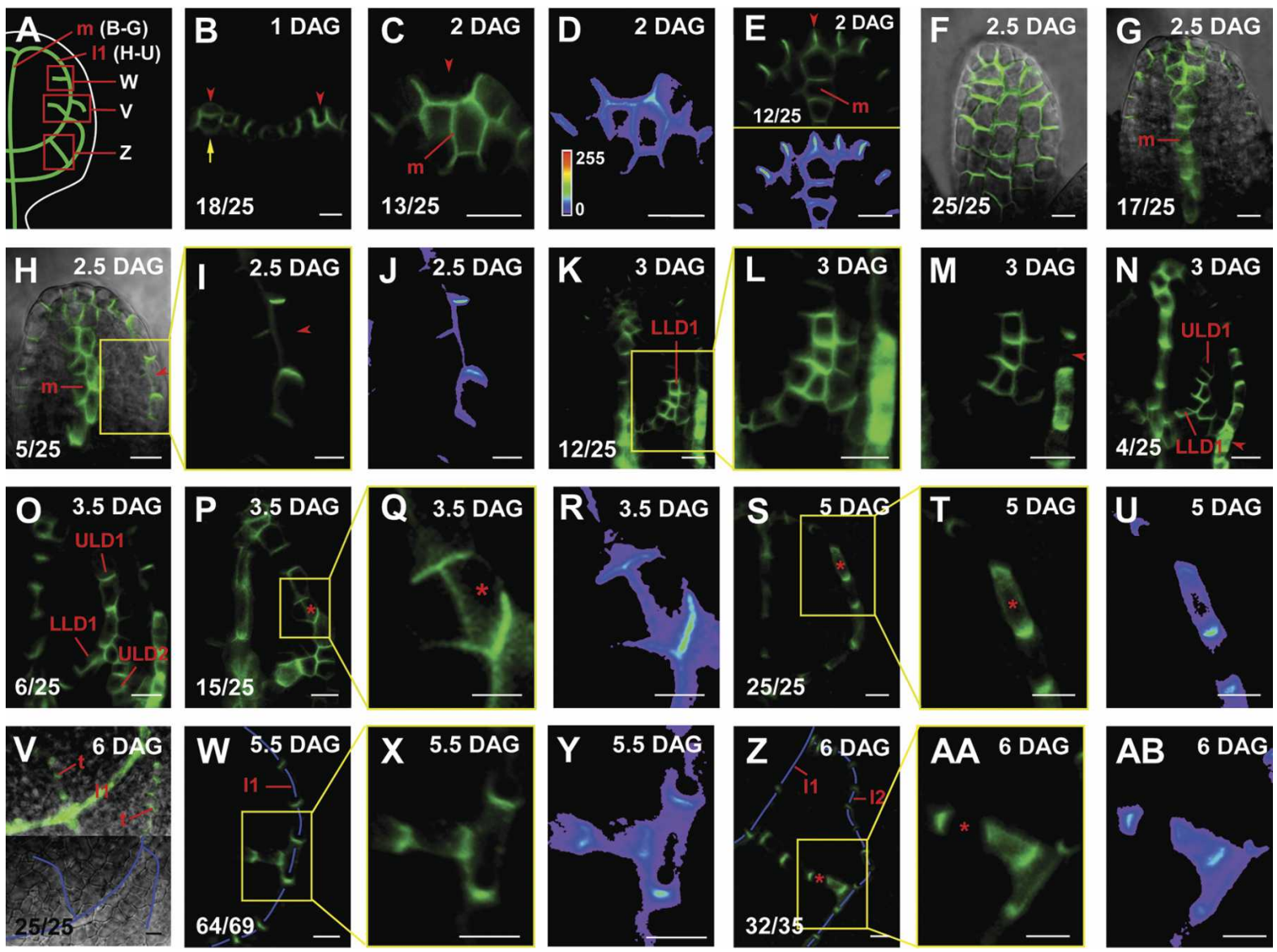

Figure 2. Acquisition of cell identity and polarity in vein formation. Arabidopsis first-leaf primordia. PIN1:GFP marker throughout. Sample orientation and all in-figure information as in Figure 1. (Red arrowhead) Convergence point; (red asterisk) bipolar cell; (m) midvein; (ULD\#, LLD\#) subdomains of vein loop number (1\#) as defined in Figure 7. (A) Schematic PED pattern of 6-DAG first leaf depicting the positions of images shown in $B-Z$. Early vein ontogenies are temporally connected in Figure 7 and summarized in Supplementary Movie S1. (B-G) Apical convergence points and midvein PED formation in young leaf primordia. Largely epidermal PIN1 expression in barely visible 1-DAG leaf primordia in $B$. Note that the cell at each primordium tip is flanked by cells of opposite polarities; furthermore, PIN1 expression beneath the tip epidermal cell in the left primordium (arrow). (C,E) Pronounced subepidermal PIN1 expression in 2-DAG primordia. LUT signal intensity monitor in $D$ and $E$ (bottom) visualize the epidermal polarity orientation toward the primordium tip, which is associated with a general tip-directed polarity in the epidermal layer at this stage $(F) .(F, G)$ Tangential abaxial $(F)$ and median $(G)$ longitudinal optical sections of the same leaf primordium. Note the apical PIN1:GFP polarity in epidermal cells $(F)$ associated with basal PIN1:GFP polarity in the midvein $(G)$. $(H-N)$ First (second-order vein) loop initiation at lateral convergence point. Convergence point formation at primordium flank $(H)$ (enlarged in $I$, signal quantified in $J$ ) followed by the formation of a broad subepidermal LLD1 domain $(K)$ (enlarged in $L$, smaller pinhole in $M$ to visualize convergence point) that becomes connected distally to the midvein by the formation of a gradually extending ULD1 (N). Note the basal PIN1 polarity in both LLD1 and ULD1 $(N, O) .(O-U)$ Completion of ULD1 formation. Integration of LLD1 and ULD1 $(O, P)$, associated with a stretch of opposite polarity distal to a bipolar cell $(P)$ (enlarged in $Q$, intensity quantified in $R$ ). $(S-U)$ Persistence of bipolar cell in fully integrated first loop. $(V-A B)$ Higher-order veins. $(V)$ Initiation of third-order vein PEDs in median optical section (top) is not associated with any epidermal PIN1 expression (tangential abaxial optical section, bottom). (W-Y) Initiation of third-order freely ending PED in the area enclosed by the first loop. Enlargement in $X$ and intensity monitored in $Y$ to visualize basal polarity both in the first loop and in the newly formed third-order PED. $(Z-A B)$ "Connected" third-order PEDs. PED connecting the first and second loops $(Z)$ comprises a bipolar cell bridging two stretches of opposite polarities, each directed toward the adjacent pre-existing PED. Enlargement $(A A)$ and intensity monitor $(A B)$ to visualize opposite polarities and bipolar cell. $(B-E, I-U, W-A B)$ GFP fluorescence. $(F-H, V)$ GFP fluorescence and transmitted light. $(I, L, Q, T, X, A A)$ Details of the areas boxed in $H, K, P, S, W$, and $Z$, respectively. $(D, E[b o t t o m], J, R, U, Y, A B) L U T$ images of $C, E$ (top), $I, Q, T, X$, and $A A$, respectively (LUT palette and corresponding gray values shown in $D$ only). Note that PIN1:GFP polarity is not visible in lower-order veins in $V$ because of the large pinhole settings used to visualize PIN1:GFP polarity in higher-order veins. Veins are outlined by blue lines in $V, W$, and $Z$. Bars: $B-H, K-P, S-A B, 10 \mu \mathrm{m} ; I, J, Q, R, 5 \mu \mathrm{m}$. Further stages and higher-resolution images are shown in Supplementary Figures S2 and S3.

rosette (below), there is a tendency toward initially broader LLDs that gradually narrow down. This process is completed by $\sim 6$ DAG (Supplementary Fig. S2), prior to the onset of Athb8 expression in the LLD3. Therefore, as most clearly documented in these later LLDs, PIN1 expression initially exceeds $A t h b 8$ expression and sites 
of procambium formation, but gradually becomes restricted toward those sites.

\section{Initiation of higher-order veins}

Vein orders 3 and higher form after the second-order loops at less predictable positions within the leaf lamina. As we did not identify any order-specific differences in their ontogeny, we refer to them collectively as higherorder veins. The PEDs of these vein orders typically appear as cell rows of single cell width in the absence of recognizable epidermal expression (Fig. 2V). Higher-order vein PEDs are never formed in isolation but are invariably continuous with earlier established PEDs or with the respective mature veins (Fig. 2W-AB; Supplementary Fig. S3). The polarity of PIN1 localization in freely ending PEDs (connected to pre-existing PEDs only at one end) is invariably basal (Fig. 2W-Y; Supplementary Fig. S3). Subcellular localization of PIN1:GFP in connected PEDs is also basal, but with regard to the preexisting PEDs at both ends. Thus, each of these PEDs is comprised of two stretches of opposite polarity, bridged by a single bipolar cell (Fig. 2Z-AB; Supplementary Fig. S3), which is typically located near one end (Figs. 2ZAB,7D [below]; Supplementary Movie S1).

In summary, higher-order vein PEDs are invariably continuous with pre-existing PIN1 expression domains and display uniform polarity toward them. Given the experimental evidence for the derivation of "connected" veins from freely ending intermediates (Scarpella et al. 2004) and the ontogeny of ULDs, it seems likely that the acquisition of a second stretch of opposite polarity is a late event associated with PED merging (Fig. 7D, below).

\section{Vein formation in higher rosette leaves}

Vein formation in leaves following the first leaf pair (higher leaves) comprises similar vein types, but those leaves have larger lamina areas, more secondary veins, and a more reticulated network of higher-order veins (Fig. 3A-C). PIN1 expression in higher leaves shows similar dynamics as described for the first leaf pair, but is typically visible in more cells than in the first leaf pair and LLDs are initially broader (Fig. 3G-I). The formation of several second-order veins can temporally overlap, such that a substantial fraction of all ground meristem cells is recruited into second-order PEDs (Fig. 3G,H). Except for the two most distal pairs, second-order veins are branched rather than loop shaped. They usually end distally in lamina protrusions associated with hydathodes or, in other cases, cannot be easily discerned close to the margin in mature leaves (Fig. 3A-C). We confirm that hydathode positions regularly coincide with strong and persisting DR5 auxin response maxima (Fig. 1F; Aloni et al. 2003), suggesting that vein formation under these conditions of generally elevated PIN1 expression may reflect the influence of higher auxin concentrations in higher leaves.

Where second-order veins do not form loops, they are nevertheless connected to each other through third-order veins along the margin (Fig. 3A-C). Strikingly, the PEDs of those second-order veins and their connecting third-
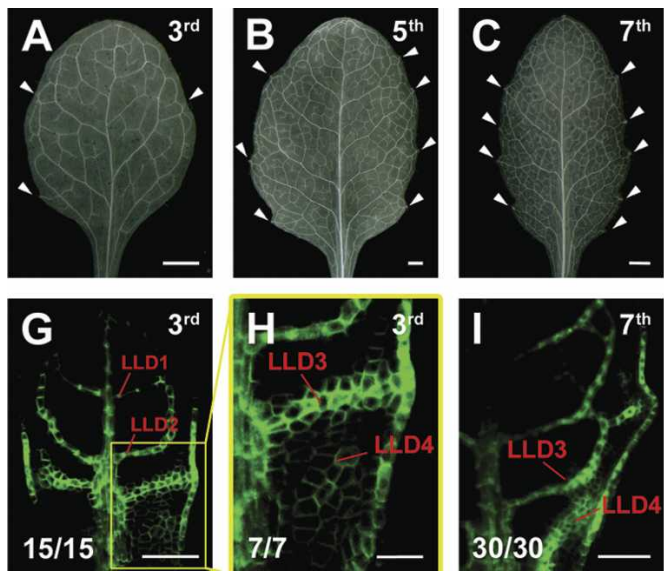
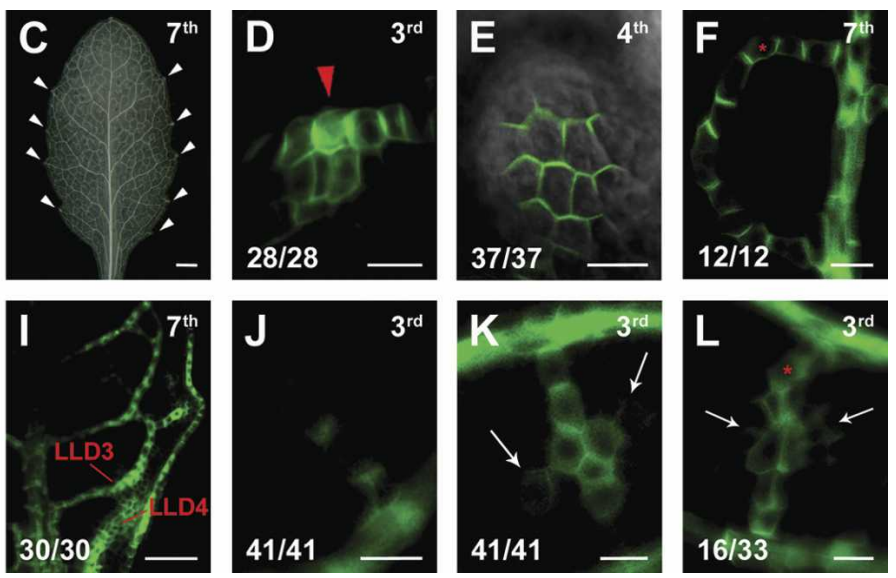

Figure 3. Higher rosette leaf features. Arabidopsis third, fourth, fifth, and seventh leaf primordium as indicated in the upper right corner of each panel. PIN1:GFP in $D-L$. Sample orientation and in-figure information as in Figures 1 and 2 . $(A-C)$ Mature venation patterns. Increased numbers of second-order veins, less integrated into loops in higher rosette leaves. Ends of major veins are typically associated with hydathodes (white arrowheads). Note the increased density and connectedness of higher-order venation in $B$ and $C$. $(D-F)$ General features. $(D)$ Convergence point-associated PED formation even prior to primordium emergence on shoot meristem. (E) Tip-directed polarity in epidermis. $(F)$ Connected first-loop PED with split polarity and bipolar cell. $(G-L)$ Specific features in higher leaves. $(G-I)$ Increased PIN1 expression and more simultaneous formation of loops. Note the broad LLD3 and fairly amorphous PIN1 expression at incipient fourth loop $(G$, enlarged in $H)$ followed by gradual LLD4 formation $(I)$. $(J-L)$ More simultaneous higher-order vein formation. (J) Narrow freely ending PED. (K) Freely ending PED with two side branches (arrows). $(L)$ Side branches (arrows) on connecting PED. Note that the side branches in $K$ and $L$ form nearly simultaneously with the respective low-order PED. (A-C) Dark-field illumination. $(D, F-L)$ GFP fluorescence. $(E)$ GFP fluorescence and transmitted light. Bars: $A-C, 1 \mathrm{~mm} ; D-F, J-L, 10 \mu \mathrm{m}$; $G, I$, $50 \mu \mathrm{m} ; H, 20 \mu \mathrm{m}$. 
Scarpella et al.

order veins are nearly perfectly reminiscent of the LLDs and ULDs of looped second-order veins (Fig. 3G-I). These observations support the notion of a composite origin of looped veins.

Like LLDs, higher-order PEDs of higher leaves are usually wider at first emergence. They are often irregularly shaped, with subcellular polarities indicating the near simultaneous emergence of third-order and branching fourth-order PEDs (Fig. 3K,L). This feature appears correlated to the tightly reticulated higher-order venation patterns in those rosette leaves (Fig. 3A-C).

In summary, PED patterning is largely similar in all rosette leaves, but in higher leaves it is associated with increased PIN1 expression and broader PEDs, and the successive appearance of different PED orders seems accelerated. These distinguishing features may reflect the influence of relatively higher auxin levels during the formation of those leaves.

\section{Vein formation in response to external auxin application}

Formation of broad PEDs, emerging near areas of apparent auxin accumulation, which then gradually narrow down as procambium formation progresses, as seen in higher leaves, is in striking agreement with mathematical models derived from the auxin canalization concept (Mitchison 1980; Rolland-Lagan and Prusinkiewicz 2005). To test whether we could mimic the effect of presumed endogenous auxin with auxin itself, we applied

Figure 4. Auxin responsiveness of PEDs and venation patterns in Arabidopsis first-leaf primordia. Primordium orientation, symbols and in-figure information as in Figures 1 and 2. Note that in this figure smaller ratios of primordia share the displayed features (lower left corner, parentheses) because only a minority of primordia responds to auxin application (Supplementary Table S2). (Yellow arrowheads) Site of IAA application. $(A, B)$ Schematic stages of primordia at time of IAA application $(A)$ and marker visualization $(B)$. (Red area) Position of IAA application. Vein color code: (brown) differentiating; (blue) procambial; (yellow) Athb8-expressing pre-procambial. $(C, D)$ DR5::GUS response to unilateral IAA $(C)$ and mock $(D)$ application. $(E-L)$ Six-DAG recording. $(F-H)$ Supernumerary convergence points $(G)$ and extremely expanded PIN1 expression $(F, G)$ in area of third loop formation. $(H)$ Intensity monitor visualizes very strong, amorphous PIN1 expression in the extremely wide PED. Note that compensating gain adjustment in $F$ results in diminished intensity of PEDs at the leaf tip. $(I-K)$ As in $F-H$, but expanded PED does not comprise all ground meristem cells and displays clear subcellular polarity (enlarged in $J$ ). $(K)$ Intensity monitor demonstrates highest PIN1 expression in the PED center. $(L)$ Formation of several, separate PEDs in the area of third loop formation. $(M-P)$ Rapid PED restriction by 7 DAG $(N-P)$ and matching features in mature leaves $(R-T)$. PED of third loop can be normal, split into multiple parallel veins $(N, O, R, S)$, or accompanied by an extra fourth loop $(P, T) .(C, D)$ DIC microscopy. $(E-P)$ GFP fluorescence. $(Q-T)$ Dark-field illumination. $(G, J)$ Details of the areas boxed in $F$ and $I$, respectively. $(H, K)$ LUT images of $G$ and $J$, respectively. Bars: $A-F, M, N, P, 50 \mu \mathrm{m}$; $G-L, O, 10 \mu \mathrm{m} ; Q, R, 500 \mu \mathrm{m} ; S, T, 100 \mu \mathrm{m}$. indole-3-acetic acid (IAA) in lanolin paste to young leaf primordia and recorded the effects on PED and vein formation. Auxin was applied distally to one side of 5-DAG primordia, but had its most dramatic effect on patterning events proximally within the newly formed areas of the leaf lamina (outlined in Fig. 4A,B).

As shown in Figure 4, auxin application results in the formation of excess numbers of convergence points in the epidermis and dramatic up-regulation and expansion of PIN1 expression in the ground meristem. This is in line with earlier reports that PIN1 expression itself is auxin responsive (Heisler et al. 2005; Vieten et al. 2005). In many cases, virtually all ground meristem cells in the vicinity of the application site express PIN1 at high levels, and as these levels subside, basal or centrobasal PIN1 polarities become apparent in individual cells (Fig. 4F-K; Supplementary Table S2). Most interestingly, subsequent decline of PIN1 expression is not uniform. Instead, PIN1 expression can become gradually restricted to multiple narrow, but continuous domains, which correlate with the formation of extra veins (Fig. 4L,N-P,R-T; Supplementary Table S2).

We conclude that auxin supply defines the formation
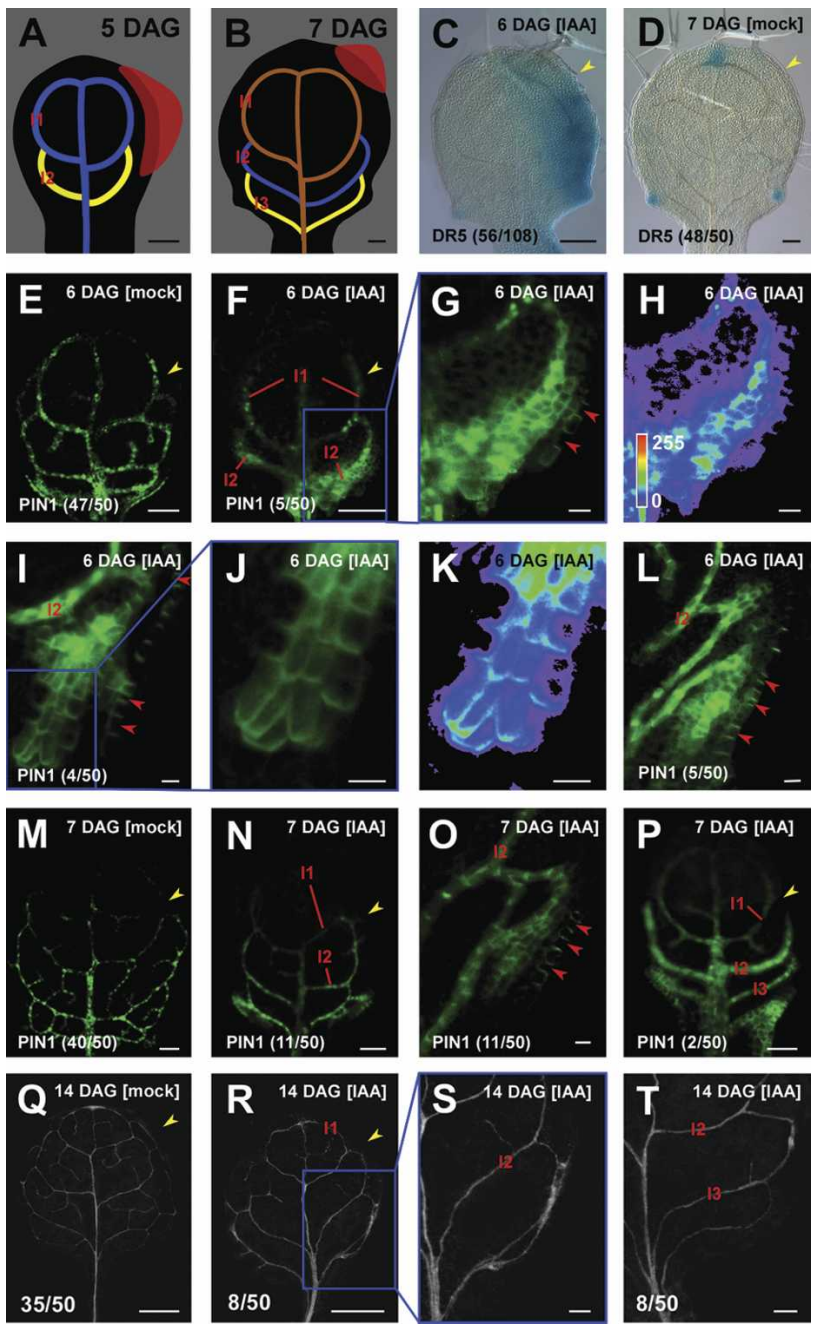
of convergence points and the initial widths of PEDs. Furthermore, PEDs have an inherent tendency to rapid self-restriction. Finally, responses to auxin application are restricted to less mature, proximal parts of the primordium. Within those responsive areas, all ground meristem cells and all epidermal cells seem to be equivalently responsive to auxin, and auxin turns out to be sufficient for the induction of new procambial strands.

\section{Vein formation under conditions of reduced auxin} transport

Progressively restricted expression of an auxin-inducible presumptive auxin-efflux protein is most readily explained by auxin drainage, feeding back on its expression. To test whether PED narrowing is auxin-transport dependent, we exposed leaf primordia to the auxin efflux inhibitor 1- $N$-naphthylphthalamic acid (NPA). As shown in Figure 5 and Supplementary Figure S4, leaf primordia with reduced auxin transport display two main characteristics. First, PEDs are typically extremely expanded (Fig. 5, cf. F-H and E). Upon NPA exposure above $10 \mu \mathrm{M}$, large areas of ground meristem cells continue to express PIN1 up to 3 DAG in a majority of first leaf primordia (cf. frequencies in Fig. 5E-H). Second, resolution of nearubiquitous PEDs into discrete domains, most clearly visible in the distal half of primordia at 4 DAG, is dependent on auxin transport (Fig. 5I-L). PED narrowing is considerably delayed at NPA concentrations above 10 $\mu \mathrm{M}$, but still results in further restricted PEDs by 5 DAG (Fig. 5M,N,P) and in only slightly enlarged veins in the mature venation pattern (Fig. 5Q-T). As with auxin application, the appearance of strong, broad PEDs is foreshadowed by an increased number of epidermal convergence points, which are already recognizable in 2- and 3-DAG primordia (Fig. 5A-H). The auxin-transport dependence of convergence point numbers demonstrates that those are also not genetically specified but subject to auxin-transport-dependent self-organization.

In summary, the dynamics of PIN1 expression in auxin-transport-inhibited leaf primordia revealed huge expansion and delayed narrowing of PEDs in conjunction with the amplification of epidermal convergence points (see Discussion).

\section{PED continuity in vascular continuity mutants}

We finally asked whether PEDs are continuous in vascular continuity mutants. Such mutants display isolated stretches of vascular strands, and their phenotypes have been considered inconsistent with signal flow through incipient veins (Carland et al. 1999; Deyholos et al. 2000; Koizumi et al. 2000). The discontinuous vein segments could, for example, have been formed in complete isolation and could therefore hint to a signal-flow-independent strand-forming mechanism. Their positions might also not coincide with those of PEDs. To assess these possibilities, we chose to visualize PEDs in the very severe vascular continuity mutant, vascular network3
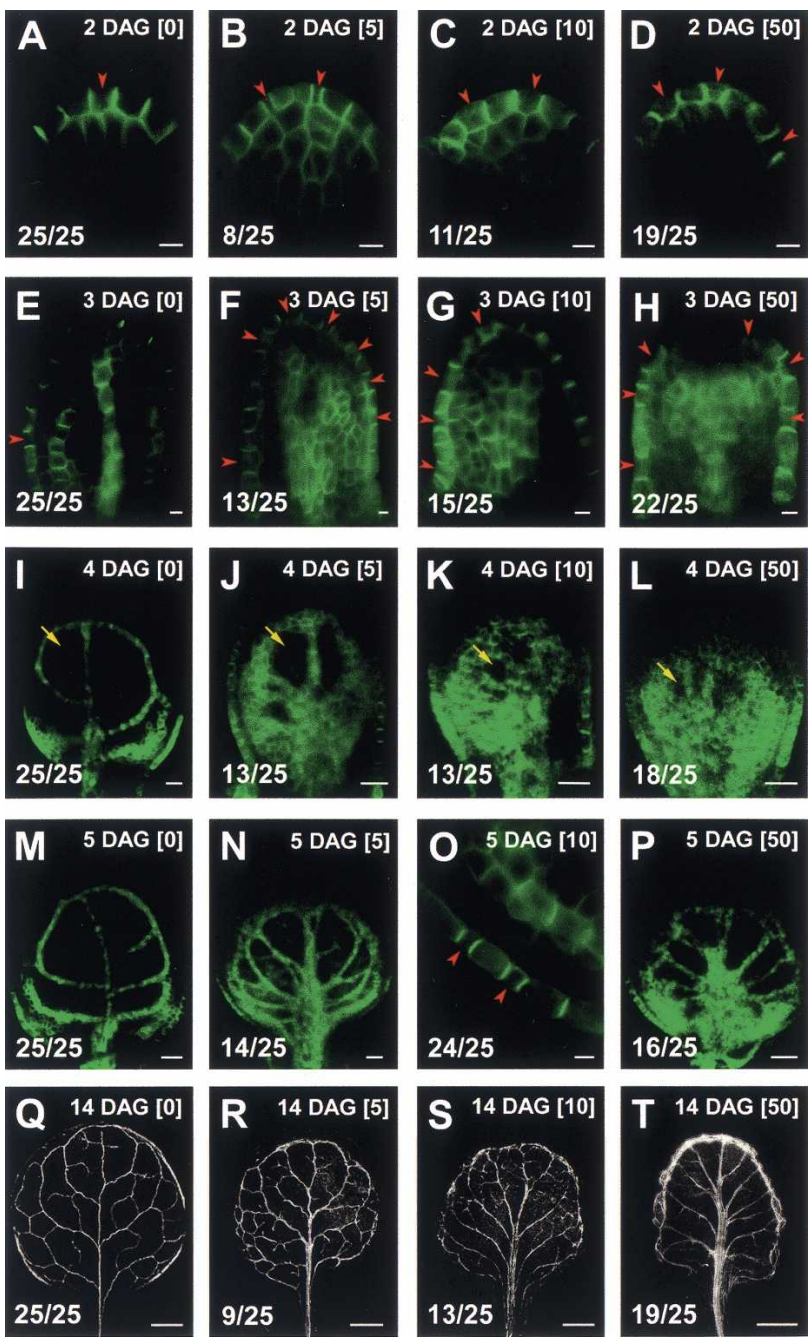

Figure 5. Auxin-transport dependence of PIN1 expression and venation patterns in Arabidopsis first-leaf primordia. NPA concentration in micromoles per liter (upper right corner) in square brackets. PIN1:GFP in $A-P$. Primordium orientation, symbols, and in-figure information as in Figures 1 and 2. $(A-D)$ Primordium initiation. Note the early appearance of supernumerary convergence points with increased NPA concentrations. $(E-P)$ NPA-dependent expansion of PEDs $(F-H, J-L, N, P)$ and presence of extra convergence points $(F-H, O)$. At 3 DAG, nearly all ground meristem cells may express PIN1 $(F-H)$, whereas from 4 DAG, PED restriction toward narrower strands is recognizable by the appearance of dark areas (arrows in $I-L$ ). Note that the size of these areas is inversely proportional to the applied NPA concentration, reflecting auxin transport dependence of PED narrowing. (Q-T) Mature venation patterns. Similarity between mature venation patterns and PEDs (see also Supplementary Fig. S4) demonstrates the congruence of PEDs with vascular differentiation zones under all experimental conditions. Note that NPA exposure delays PED narrowing, but does not prevent it. Mature veins are narrow under all experimental conditions. $(A-P)$ GFP fluorescence. $(Q-T)$ Dark-field illumination. Bars: $A-H, O, 5 \mu \mathrm{m} ; I-L, 20 \mu \mathrm{m} ; M, N, P, 50 \mu \mathrm{m}$; Q-T, $500 \mu \mathrm{m}$.

(van3). In van3 mutants, discontinuity has been demonstrated even for the pre-procambial Athb8 expression do- 
Scarpella et al.

mains, indicating that continuity is already distorted prior to any overt acquisition of vascular cell fates (Koizumi et al. 2000).

As shown in Figure 6, lower-order PEDs in van3 mutants are essentially continuous at their inception (Fig. $6 \mathrm{H}, \mathrm{I}, \mathrm{K})$. The continuity of PEDs is contrasted by the subsequent discontinuity of Athb8 expression domains (Fig. $6 \mathrm{~B}, \mathrm{D}, \mathrm{F})$, but the positions of midvein and second-order veins coincide with those of PEDs and can be considered as isolated fragments of initially continuous zones outlined by PIN1 expression. Remarkably, van3 mutants also show the normal sequence of PED initiations, including their origin near epidermal convergence points and the successive formation of second-order vein loops and higher-order venation, revealing an amazingly normal early vein ontogeny (Fig. 6H,I,K,L). However, PED continuity is fragile in van3 mutants, leading to discontinuous second-order PEDs in all loops beginning at 4 DAG (Fig. 6K,L).

Because higher-order veins occur at highly variable positions, using our methods, they cannot individually be monitored shortly after their initiation. Nevertheless, the fact that the percentage of continuous higher-order veins is highest at their inception at 4 DAG and then continuously decreases suggests an ontogeny similar to that of lower-order PEDs (Fig. 6L,N-P). The fragmented PEDs are not without substructure. Instead, subcellular PIN1 polarity seems to be directed toward the brightest cells within a PED in cell clusters that typically are amorphous rather than extended (Fig. 6N-P). These PIN1-expressing cell clusters correspond to amorphous areas of Athb8::GUS expression (Fig. 6F).

In summary, perfect alignment of PEDs with vascular differentiation zones in the van3 mutant indicates that the primary defect lies in the maintenance rather than in the initial establishment of a continuous vascular pattern. The fact that the onset of fragmentation is reflected already in a gradual decay of the PIN1 expression and polarity pattern is consistent with an involvement of the VAN3 ARF-GAP in promoting vascular continuity through PIN genes.

\section{Discussion}

Among the patterning mechanisms in which auxin signal flow has been implicated (summarized in Leyser
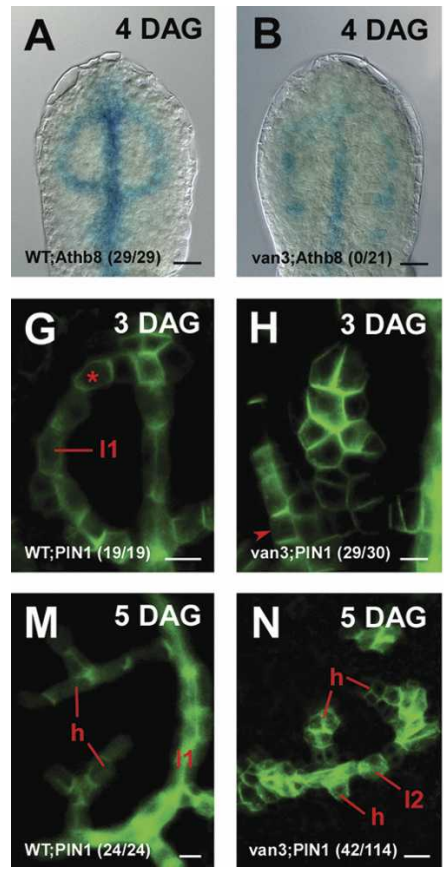
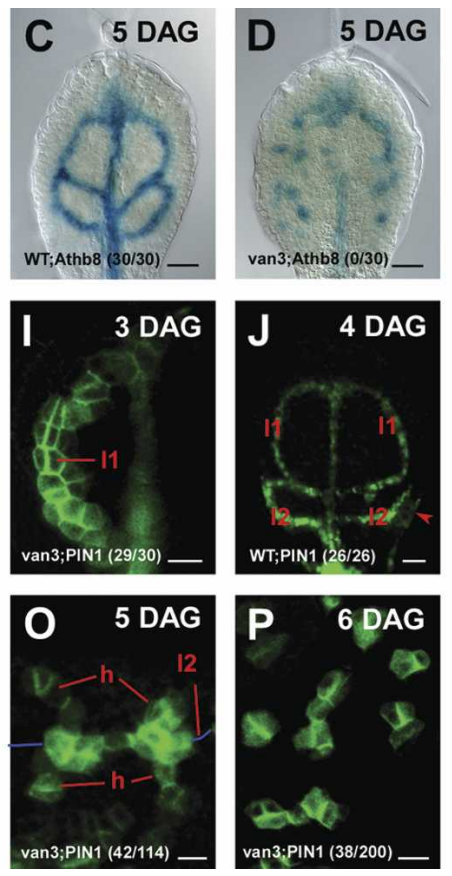
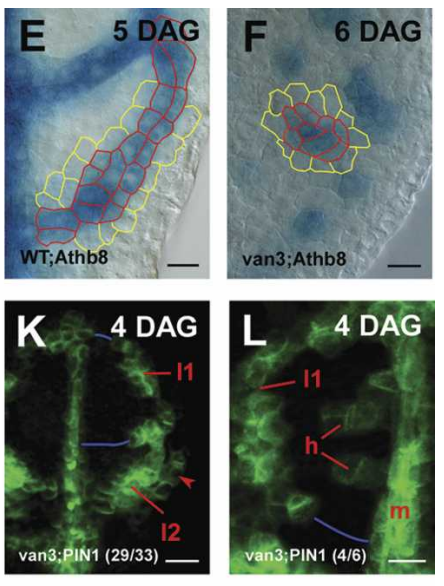

Figure 6. Continuous vascular cell fate specification in vascular continuity mutant. Arabidopsis first-leaf primordia. Fractions in the lower left corner reflect proportions of continuous marker expression domains/veins in each genotype (Supplementary Table S1). Sample orientation and all in-figure information as in Figures 1 and 2. Fragmented vein segments are connected by blue lines. $(A-F)$ Pre-procambial cell fate acquisition (marked by Athb8::GUS expression) in wild-type $(A, C, E)$ and van3 $(B, D, F)$ leaves. Athb8::GUS expression is generally faint and discontinuous in van3 mutants. The cell boundaries outlined in $E$ and $F$ demonstrate that preprocambial cells cannot be distinguished by cell shape from ground meristem cells (yellow) at the displayed stages. Note that at 6 DAG, pre-procambium arises in clusters rather than veins in van3 mutants. $(G-I)$ Convergence point-initiated LLD1 formation in $\operatorname{van} 3(H)$ leading to closed loop $(I)$ as in wild type $(G)$. $(J-K)$ Equivalently positioned second-loop initiation in wild type $(J)$ and van3 $(K)$. Note the discontinuous first loop in $K .(L-P)$ Faint, heterogeneous, but largely continuous, PIN1:GFP expression in early higher-order (h) PEDs $(L)$, followed by increasingly heterogeneous $(N, O)$ and entirely disconnected $(P)$ PIN1 expression on the following days. By 6 DAG and later, the van3 PEDs $(P)$ and subsequent xylem differentiation zones $(R)$ have become entirely fragmented. $(A-F)$ DIC microscopy. $(G-P)$ GFP fluorescence. (Q-R) Dark-field illumination. Bars: A, B,J,K, $20 \mu \mathrm{m} ; C, D, 50 \mu \mathrm{m} ; E-G, I, L-P, 10 \mu \mathrm{m} ; H, 5 \mu \mathrm{m} ; \mathrm{Q}, R$, $500 \mu \mathrm{m}$. 
2005), leaf venation patterns are particularly suited for testing and refining of patterning models. They are landmark-rich, display growth-stage and species-specific variations, and have become subject to increasingly precise mathematical simulation (Mitchison 1980; Kramer 2004; Rolland-Lagan and Prusinkiewicz 2005; Runions et al. 2005). To dissect events associated with the selection of procambial cells from the leaf ground meristem, we have visualized apparent routes of auxin flow and associated cellular polarities during normal and experimentally altered leaf vascular patterning. We show that PIN1 expression precedes and converges toward sites of procambium specification and that its subcellular polarity strongly indicates auxin transport toward pre-existing vasculature. Intriguingly, we find that the positioning of the midvein and of second-order veins, which together set up the grid for reiterative higher-order vein patterning, is associated with epidermal polarity convergence points. The finding indicates a common mechanistic basis for defining reference points in leaf and shoot meristem patterning (Reinhardt et al. 2003). Our results highlight the importance of auxin-flow continuity and convergence to narrow routes as these are observed even under experimentally or genetically altered conditions and provide strong support for the notion of procambium formation along routes of sustained auxin transport. Moreover, this first detailed picture drawn by the reproducible expression of PIN1 provides clues as to how to reconcile signal-flow patterning with the formation of closed vascular networks.

\section{Subcellular PIN1 polarities in different vein orders}

Expression profiles identify PIN1 as the most important member of the PIN gene family in leaf procambium formation. Reported compensatory misexpressions of other PIN genes (Blilou et al. 2005; Vieten et al. 2005) may buffer the severity of the pin1 mutant phenotype, but do not diminish its unique role in the wildtype background. As determined in huge numbers of confocal optical sections, the subcellular polarities of PIN1:GFP in the formation of various vein orders are remarkably reproducible. Given the vast numbers of participating cells, leaf pre-procambial development displays an unprecedented degree of ordered cell polarization, strongly supporting its functional relevance in vein formation.

The basic rules of PIN1 polarity in all veins are remarkably simple. Generally, PIN1:GFP is localized to the side of cells facing pre-existing vasculature, the presumed sinks of auxin flow. Therefore, in freely ending PEDs, there is a single polarity along the entire domain (Fig. 2). Also in connected PEDs, polarity is strictly regulated and is generally directed toward pre-existing vasculature. Connected PEDs consist of no more than two subdomains of antagonistic, outwardly polarized cells (Fig. 2). In short, polarity in PEDs of all orders is directed toward pre-existing vasculature, and whether it is uniform or bidirectional is entirely dependent on the vein's connectedness to other veins.

\section{Freely ending and connected veins}

The positions of PEDs and the subcellular localization of PIN1:GFP toward the sink pole of developing freely ending veins is consistent with general concepts of auxinflow-driven vascular patterning (Sachs 1989). Moreover, initiation of PEDs at pre-existing veins and their subsequent extension away from them, opposite to the direction of auxin flow, is in line with a recent mathematical simulation of the same concept, which predicts vein initiation away from the sink (Rolland-Lagan and Prusinkiewicz 2005; e.g., see Fig. 7C, ULD extension from LLD).

Leaf venation patterns, however, do not only consist of freely ending veins, but also comprise connected veins and closed loops (Fig. 7A). Reconciling closed vein networks with a polar flow model has been subject to debate, and even recent, otherwise highly accurate simulations of natural venation patterns are specifically challenged when simulating the multitude of connections within networks (Runions et al. 2005). This is not surprising, because modeling had typically been based on the final products, the mature venation patterns, and could not take into account critical parameters of early vein ontogeny. Analyzing vein formation at the PED level illuminates the cellular environment in which veins become connected, including critical features, such as numbers, polarities, and presumptive auxintransport properties of participating cells. First, connected PEDs comprise two stretches of opposite polarity. Second, PEDs become connected early and within areas of very few cells (Figs. 2Z, 7D). Third, while PEDs are subsequently extended by intercalation (Fig. 7E), subcellular polarities within them are maintained. Finally, the development of the ULDs together with earlier observations (Scarpella et al. 2004) demonstrate that connected veins can originate from freely ending veins. The most likely scenario derived from these four observations is that within fields of surprisingly few cells, extending PEDs come in close proximity to other PEDs with correspondingly high probability (Fig. 7D). As PED extension is driven by their function as signal sinks, there should then be single cells exposed to two opposite sinks, while still receiving signal from other neighboring cells. This mechanism is formally equivalent to polarity acquisition of intercalating cells during PED extension (Fig. 7D,E), but involves PIN1 localization to opposite poles within the same cell. Such bipolar cells are observed in connected veins with high regularity, suggesting that bipolar PIN1 localization is a cell biological prerequisite for the formation of closed vascular networks.

\section{Formation of closed loops}

Pronounced loops of second-order veins are hallmarks of dicot leaf venation patterns and can be considered as a conspicuous type of connected veins. We show that at the PED level these closed loops can be resolved to be of composite origin, with each of the two constituents falling into a more general category. This origin is, in fact, recognizable in the mature pattern of higher leaves, 
Scarpella et al.

Figure 7. Conceptual summary and model interpretation (cf. animated vein ontogeny in Supplementary Movie S1). (A) Definition of vein orders (first, cyan; second, pink; higher, purple), loop numbers (11-13, each derived from corresponding LLD and ULD), freely ending (f) and connected (c) veins, hydathode (h), and proximo-distal positions as used in the text. Simplified schemes of real Arabidopsis venation pattern (left) and of most basic signal-flow prediction (right). Note that according to the auxin signal flow concept, a freely ending PED extends from its point of attachment to pre-existing veins into the dispersed source areas. $(B-D)$ PIN1-reported auxin-flow routes (green) and orientations (red arrows), and zones of pre-procambial (blue) and procambial (brown) cell identity acquisition. Three major additions to the basic concept are derived from pre-procambial observations. First, convergence point formation: Patterning in the ground meristem is externally triggered by auxin convergence in the epidermis (orange in $B, C$ ). Polarity rever-

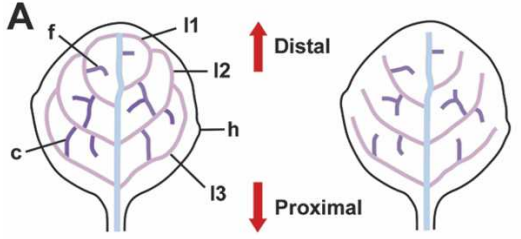

B: midvein formation

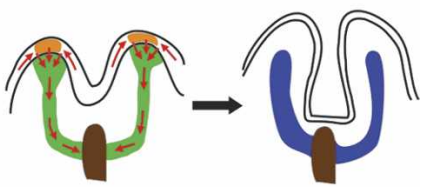

C: loop formation

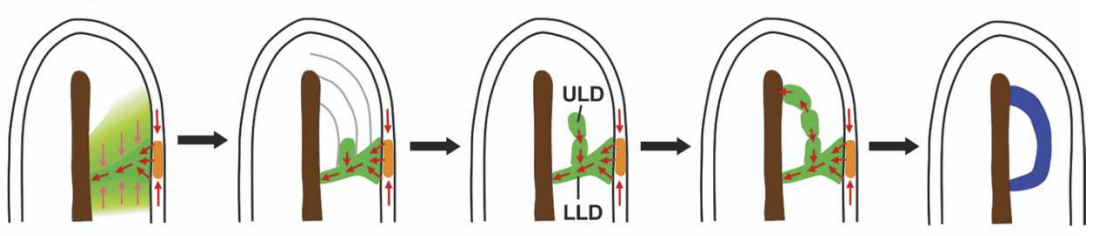

D: 'connected' vein formation

E
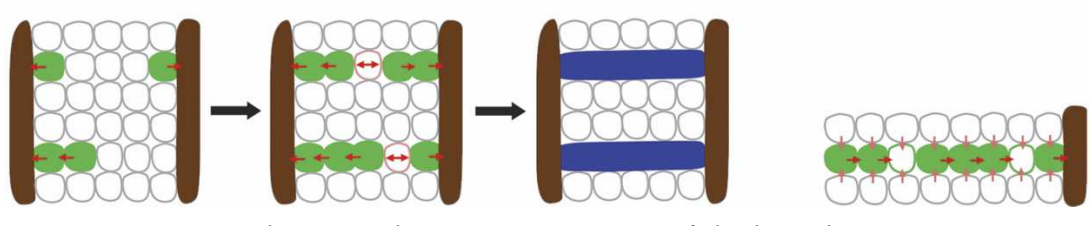

sal (Heisler et al. 2005) at the terminal convergence point may contribute to subsequent positioning of the lateral convergence points (shown in C). Auxin internalization at convergence points initiates midvein and LLD formation in extremely small primordia. Second, connected vein formation. PEDs become "connected" when cell numbers are very small (shown in $D$ ). This increases the probability that ground meristem cells are exposed to the sink effect of two PEDs (pink-outlined cells with double arrows, $D$ ) and become bipolar cells (cf. E). Third, closed terminal loop. Especially in very small primordia, the ground meristem cells are arranged in defined cell layers (gray lines in $C$ ), which seems to predispose signal-flow routes. (E) PED extension by intercalation. Note that all cells are expected to act as signal conduits (pink arrows in $C$, left, and in $E$; not drawn in other panels) toward sinks (green PEDs, red arrows). Therefore, any cell inserted into a PED by rapid cell divisions (green-outlined cells) would very quickly adopt position-dependent polarities and auxin-transport properties, thereby maintaining PED continuity. Note that the same mechanism accounts for bipolar cell formation in $C$ and $D$.

where ULDs represent typical third-order connected veins (Kang and Dengler 2004). Only in the most distal part of a leaf, this origin is obscured by their amalgamation with LLDs to form perfectly continuous loops (Fig. 7C). The ULD located directly at the leaf tip is unusually curved toward the midvein, but this corresponds to unusually curved ground meristem cell arrangements in this area (Mattsson et al. 1999), which are likely to influence auxin-transport routes (Fig. 7C). The LLD, in turn, shares many characteristics with the midvein PED, and those can be collectively classified as convergence point-associated PEDs. In the emerging picture, therefore, all Arabidopsis vein types can be generated by two basic ontogenies: convergence point-associated PEDs (midvein and LLDs) and internally initiated PEDs (ULDs and higher-order PEDs). The latter are initially freely ending, but can become connected upon proximity to other PEDs.

\section{PEDs and auxin}

Elevated auxin levels regularly lead to expanded PEDs. This is most directly visible upon auxin application, but is most likely also true in auxin-transport-inhibited primordia or, naturally, in rosette leaf primordia 3 and higher. Interestingly, expanded PIN1 expression domains narrow to one or few cell files at stages prior to the expression of the earliest pre-procambial marker
Athb8::GUS, consistent with the notion that vasculature is formed in the core areas of gradually restricted domains of elevated auxin transport (Sachs 1981; Rolland-Lagan and Prusinkiewicz 2005). This interpretation is further supported by the subcellular localization of PIN1:GFP. While PIN1:GFP polarity may not be strictly basal across wide PEDs, it is usually basal in the PED center and is strictly basal once the PED has become narrow (Figs. 2-5; Supplementary Figs. S2, S4).

Most importantly, auxin-application-induced PEDs narrow down quickly. When IAA is applied to distallateral positions in 5-DAG primordia, all ground meristem cells in a proximal responsive region transiently express PIN1, but the initially broad PED resolves to one or more narrow domains within a day. These experiments identify auxin as a sufficient signal for the induction of PIN1 expression in all ground meristem cells and for the formation of entirely new veins. They further enable visualization of the predicted involvement of all surrounding ground meristem cells, which normally express PIN1 too weakly to be recorded. Upon auxin application, PIN1 expression and polarity in these ground meristem cells are consistent with their involvement in a patterning process that competitively selects a preferred route of auxin transport. The restriction of the observed response to the proximal region demonstrates that the patterning process is limited to ground meristem cells. At the time of auxin application, ground meristem cells 
are found in the subepidermal tissue of the proximal part of the primordia, while subepidermal cells in the distal part have begun to acquire mesophyll characteristics (Pyke et al. 1991; Scarpella et al. 2004). Finally, the dependence of PED narrowing on auxin transport is indicated by their delayed restriction in NPA-treated leaf primordia (Fig. 5; Supplementary Fig. S4), where their size is remarkably proportional to the degree of auxin transport inhibition.

\section{Epidermal control of vein positions}

The involvement of the epidermis in the specification of first- and second-order veins has intriguing implications. These veins define the basic architectures of dicotyledoneous venation patterns and, through epidermal convergence points, are tightly linked to processes that set up the leaf and its shape. It is conceivable that through auxin-independent genetic control of convergence point positions, divergent venation patterns could be rigidly specified in various species. In Arabidopsis, however, our results suggest that, within defined limits (below), convergence point positioning is a self-organizing auxintransport-dependent process. Interestingly, we found convergence points to be confined to the epidermis at the leaf margin. Furthermore, also within the margin, the competence window for convergence point formation in response to applied auxin is restricted to the most immature part of the primordium. This indicates that the self-organizing process positioning convergence points is integrated into a larger developmental context, through which it is restricted to defined areas.

A self-organizing process selecting discrete epidermal spots within a defined responsive zone bears striking resemblance to the proposed mechanism for leaf positioning in the shoot meristem (Reinhardt et al. 2003). Restricted to the peripheral zone of the meristem, auxin convergence and internalization at discrete spots in the epidermis are thought to trigger processes in subepidermal tissues that include cell proliferation (leading to primordium growth) and vascular strand (leaf trace) formation (Fig. 2B; Reinhardt et al. 2003). Just as for convergence points in leaves, auxin overload in shoot meristems reduces lateral spacing and eventually leads to primordium fusion (Reinhardt et al. 2000). A similar picture is seen in embryos, where NPA treatment leads to fused cotyledons with multiple midveins (Liu et al. 1993). From this, it has been postulated that internalization-mediated auxin depletion, likely together with polarity reversal events in the epidermis (Heisler et al. 2005), constitutes the cell biological basis for the lateral inhibition mechanism that ensures proper phyllotactic spacing. By analogy, second-order veins could be appropriately spaced, because each newly formed PED acts as a sink locally draining auxin from the epidermis (Fig. $7 \mathrm{~B}, \mathrm{C})$. Alternatively, or more likely in addition, dynamic processes within the epidermis margin itself may lead to local auxin accumulation. These dynamic processes seem to involve coordinated, nonlinear responses of epidermal cells to auxin, since internalization and/or polar- ity reversals are restricted to sharply defined areas and stages. The cell-biological basis of those responses is still rather elusive, but if they involve positive feedback to auxin accumulation, it is plausible that auxin overload leads to a reduced spacing of such focal spots. Auxin has long been postulated to act as a fairly universal coordinating signal, whose precise informational content is dependent on the developmental context. The involvement of convergence points in major vein formation is an impressive example of such context modulation. If convergence point patterning in leaves and shoot meristems shares a common cell-biological basis, its mechanistic dissection may benefit from the simple, one-dimensional arrangement of margin cells around the leaf circumference.

\section{Materials and methods}

Plant material and growth conditions

The origins of all the marker lines are described in Supplementary Table S3. The SALK line 069166 (Alonso et al. 2003) carries a T-DNA insertion downstream of position +222 (i.e., position $42,161,799$ of chromosome 5) of the VAN3 gene (At5g13300) (Koizumi et al. 2005). Insertion was confirmed by genomic PCR with a VAN3 gene-specific primer $\left(5^{\prime}\right.$-TCGTTTATGGCGTCT GTGTGAGA-3') and the LBb1 primer (5'-GCGTGGACCGCT TGCTGCAACT-3').

In all experiments, seeds were surface sterilized, synchronized, and germinated on growth medium at the approximate density of $1 \mathrm{seed} / \mathrm{cm}^{2}$ as described (Scarpella et al. 2004). Sealed plates were incubated at $25^{\circ} \mathrm{C}$ under continuous fluorescent light $\left(100 \mu \mathrm{E} \mathrm{m} \mathrm{m}^{-2} \mathrm{sec}^{-1}\right)$. We define "days after germination" (DAG) as days after exposure of imbibed seeds to light. To study the development of leaf 3 and higher, which we collectively refer to as "higher leaves," 2-DAG seedlings were transferred to growth medium in Magenta GA-7 vessels (Sigma) at the approximate density of 0.25 seedling $/ \mathrm{cm}^{2}$ and grown at $25^{\circ} \mathrm{C}$ under continuous fluorescent light $\left(100 \mu \mathrm{E} \mathrm{m} \mathrm{m}^{-2} \mathrm{sec}^{-1}\right)$.

\section{Auxin application}

For local auxin treatments of first-leaf primordia, IAA (Sigma) at $10 \%(\mathrm{w} / \mathrm{v})$ (final concentration) was dissolved in a prewarmed paste consisting of lanolin (Sigma) and a liposoluble red dye (Procter \& Gamble). The paste was manually applied to one side of one first-leaf primordium of each 5-DAG seedling with insect pins (0.1 mm diameter; Fine Science Tools, Inc.) and an ophthalmic surgical pin holder (Fine Science Tools, Inc.). The red dye in the lanolin paste allowed the recognition of the application site on each primordium. Control 5-DAG leaf primordia were mock-treated with the red lanolin paste only.

\section{Auxin transport inhibition}

To study the effects of auxin transport inhibition on first-leaf development, seeds were germinated on growth medium supplemented with defined concentrations of 1-N-naphthylphthalamic acid (NPA; Chem Service). Thus, first-leaf primordia were exposed to NPA $1 \mathrm{~d}$ before they became visible, which occurs at 1 DAG (Fig. 2B). Third-leaf primordia became visible at 3 DAG (Fig. 3D). Therefore, for auxin transport inhibition treatments of third-leaf primordia that were comparable to those performed on first-leaf primordia, 2-DAG seedlings were transferred to growth medium supplemented with defined NPA 
concentrations. For even NPA exposure, seedlings were submerged in a 2-mL film of liquid growth medium supplemented with the same NPA concentration present in the solid growth medium.

\section{Microtechniques and microscopy}

Histochemical detection of GUS activity, fixation, and clearing were performed as described (Scarpella et al. 2004). Detailed staining conditions are provided in Supplementary Table S4. Samples were viewed with a Leica MZFLIII stereomicroscope (Leica Microsystems) equipped with a Canon EOS D60 digital camera (Canon, Inc.), with a Zeiss Axio Imager.Z1 microscope equipped with an AxioCam HRm digital camera (Carl Zeiss), or with an Olympus BX51 microscope (Olympus Optical Co.) equipped with a Photometrix CoolSnap fx digital camera (Roper Scientific) and a MicroColor liquid crystal tunable RGB filter (Cambridge Research \& Instrumentation, Inc.).

For confocal laser scanning microscopy, dissected leaf primordia were mounted in water and observed with a Zeiss Axiovert 100M microscope equipped with a Zeiss LSM510 laser module confocal unit (Carl Zeiss). GFP was visualized with the 488-nm line of an Argon laser at $50 \%-55 \%$ of output and $4 \%-10 \%$ transmission, and with either a 505-530- or a 500-550-nm bandpass filter. Signal-to-noise ratio was increased during image acquisition by four-frame averaging (Russ 1995), and post-acquisition by neighborhood averaging with a $9 \times 9$ Gaussian kernel with a standard deviation radius of 1 pixel (Russ 1995; van Kempen et al. 1997). Because the human eye cannot distinguish all 256 different levels of brightness (Russ 1995; Young et al. 1998), to increase perception of monochromatic shades, and thus convey the spectral detection range under which the data were acquired, critical fluorescence images were turned into 8-bit gray-scaled images and pseudocolored with the rainbow spectrum look-up-table (LUT) of ImageJ (National Institutes of Health, http://rsb.info.nih.gov/ij).

All images were assembled using Adobe Photoshop 7.0 (Adobe Systems), and figures were labeled using Canvas X (ACD Systems Ltd.).

\section{Acknowledgments}

We thank Simona Baima, Giorgio Morelli, Jane Murfett, Tom Guilfoyle, and Cold Spring Harbor Laboratory for seeds; Mike Deyholos and Andrew Waskiewicz for allowing the use of their microscopy equipment; and Nancy Dengler and Koji Koizumi for helpful comments on the manuscript. This work was supported by Discovery Grants of the Natural Sciences and Engineering Research Council of Canada (NSERC) (to T.B. and E.S.), by an Alberta Ingenuity New Faculty Grant (to E.S.), by the Canada Research Chairs Program (to E.S.), and by an Ontario Premier's Research Excellence Award (to T.B). D.M. was supported by an NSERC PGS-M fellowship.

\section{References}

Aloni, R., Schwalm, K., Langhans, M., and Ullrich, C.I. 2003. Gradual shifts in sites of free-auxin production during leafprimordium development and their role in vascular differentiation and leaf morphogenesis in Arabidopsis. Planta 216: $841-853$.

Alonso, J.M., Stepanova, A.N., Leisse, T.J., Kim, C.J., Chen, H., Shinn, P., Stevenson, D.K., Zimmerman, J., Barajas, P., Cheuk, R., et al. 2003. Genome-wide insertional mutagenesis of Arabidopsis thaliana. Science 301: 653-657.
Benkova, E., Michniewicz, M., Sauer, M., Teichmann, T., Seifertova, D., Jurgens, G., and Friml, J. 2003. Local, efflux-dependent auxin gradients as a common module for plant organ formation. Cell 115: 591-602.

Blilou, I., Xu, J., Wildwater, M., Willemsen, V., Paponov, I., Friml, J., Heidstra, R., Aida, M., Palme, K., and Scheres, B. 2005. The PIN auxin efflux facilitator network controls growth and patterning in Arabidopsis roots. Nature 433: 3944.

Carland, F.M., Berg, B.L., FitzGerald, J.N., Jinamornphongs, S., Nelson, T., and Keith, B. 1999. Genetic regulation of vascular tissue patterning in Arabidopsis. Plant Cell 11: 21232137.

Deyholos, M.K., Cordner, G., Beebe, D., and Sieburth, L.E. 2000. The SCARFACE gene is required for cotyledon and leaf vein patterning. Development 127: 3205-3213.

Esau, K. 1965. Plant anatomy. John Wiley, New York.

Foster, A.S. 1952. Foliar venation in angiosperms from an ontogenetic standpoint. Am. J. Bot. 39: 752-766.

Heisler, M.G., Ohno, C., Das, P., Sieber, P., Reddy, G.V., Long, J.A., and Meyerowitz, E.M. 2005. Patterns of auxin transport and gene expression during primordium development revealed by live imaging of the Arabidopsis inflorescence meristem. Curr. Biol. 15: 1899-1911.

Kang, J. and Dengler, N. 2004. Vein pattern development in adult leaves of Arabidopsis thaliana. Int. J. Plant Sci. 165: 231-242.

Koizumi, K., Sugiyama, M., and Fukuda, H. 2000. A series of novel mutants of Arabidopsis thaliana that are defective in the formation of continuous vascular network: Calling the auxin signal flow canalization hypothesis into question. Development 127: 3197-3204.

Koizumi, K., Naramoto, S., Sawa, S., Yahara, N., Ueda, T., Nakano, A., Sugiyama, M., and Fukuda, H. 2005. VAN3 ARFGAP-mediated vesicle transport is involved in leaf vascular network formation. Development 132: 1699-1711.

Kramer, E.M. 2004. PIN and AUX/LAX proteins: Their role in auxin accumulation. Trends Plant Sci. 9: 578-582.

Leyser, O. 2005. Auxin distribution and plant pattern formation: How many angels can dance on the point of PIN? Cell 121: 819-822.

Liu, C., Xu, Z., and Chua, N.H. 1993. Auxin polar transport is essential for the establishment of bilateral symmetry during early plant embryogenesis. Plant Cell 5: 621-630.

Mattsson, J., Sung, Z.R., and Berleth, T. 1999. Responses of plant vascular systems to auxin transport inhibition. Development 126: 2979-2991.

Meinhardt, H. 1982. Models of biological pattern formation. Academic Press, London.

Mitchison, G.J. 1980. Model for vein formation in higher-plants. Proc. R. Soc. Lond. B. Biol. Sci. 207: 79-109.

Nelson, T. and Dengler, N. 1997. Leaf vascular pattern formation. Plant Cell 9: 1121-1135.

Okada, K., Ueda, J., Komaki, M.K., Bell, C.J., and Shimura, Y. 1991. Requirement of the auxin polar transport system in early stages of Arabidopsis floral bud formation. Plant Cell 3: 677-684.

Pyke, K.A., Marrison, J.L., and Leech, R.M. 1991. Temporal and spatial development of the cells of the expanding 1st leaf of Arabidopsis-thaliana (L) Heynh. J. Exp. Bot. 42: 1407-1416.

Reinhardt, D., Mandel, T., and Kuhlemeier, C. 2000. Auxin regulates the initiation and radial position of plant lateral organs. Plant Cell 12: 507-518.

Reinhardt, D., Pesce, E.R., Stieger, P., Mandel, T., Baltensperger, K., Bennett, M., Traas, J., Friml, J., and Kuhlemeier, C. 2003. Regulation of phyllotaxis by polar auxin transport. Nature 
426: $255-260$.

Rolland-Lagan, A.G. and Prusinkiewicz, P. 2005. Reviewing models of auxin canalization in the context of leaf vein pattern formation in Arabidopsis. Plant J. 44: 854-865.

Runions, A., Fuhrer, M., Lane, B., Federl, P., Rolland-Lagan, A.-G., and Prusinkiewicz, P. 2005. Modeling and visualization of leaf venation patterns. ACM Trans. Graph. 24: 702711.

Russ, J.C. 1995. The image processing handbook. CRC Press, Boca Raton, FL.

Sachs, T. 1981. The control of the patterned differentiation of vascular tissues. Adv. Bot. Res. 9: 151-262.

. 1989. The development of vascular networks during leaf development. Curr. Top. Plant Biochem. Physiol. 8: 168183.

1991. Cell polarity and tissue patterning in plants. Development. Suppl. 1: 83-93.

Scarpella, E., Francis, P., and Berleth, T. 2004. Stage-specific markers define early steps of procambium development in Arabidopsis leaves and correlate termination of vein formation with mesophyll differentiation. Development 131: 3445-3455.

Turner, S. and Sieburth, L. 2002. Vascular patterning. In The Arabidopsis book (eds. C.R. Somerville and E.M. Meyerowitz). American Society of Plant Biologists, Rockville, MD. doi/10.1199/tab.0073; http://www.aspb.org/publications/ arabidopsis.

van Kempen, G.M.P., van Vliet, L.J., and Verveer, P.J. 1997. Application of image restoration methods for confocal fluorescence microscopy. In Three-dimensional microscopy: Image acquisition and processing IV. Proceedings of SPIE vol. 2984. (eds. C.J. Cogswell et al.), pp. 114-124. SPIE-The International Society for Optical Engineering, Bellingham, WA.

Vieten, A., Vanneste, S., Wisniewska, J., Benkova, E., Benjamins, R., Beeckman, T., Luschnig, C., and Friml, J. 2005. Functional redundancy of PIN proteins is accompanied by auxin-dependent cross-regulation of PIN expression. Development 132: 4521-4531.

Wisniewska, J., Xu, J., Seifertová, D., Brewer, P.B., Ruzicka, K., Blilou, I., Benková, E., Scheres, B., and Friml, J. 2006. Polar PIN localization is sufficient to direct auxin flow in plants. Science (in press).

Young, I.T., Gerbrands, J.J., and van Vliet, L.J. 1998. Fundamentals of image processing. In The digital signal processing handbook (eds. V.K. Madisetti and D.B. Williams), pp. 51.01-51.81. CRC Press, Boca Raton, FL. 


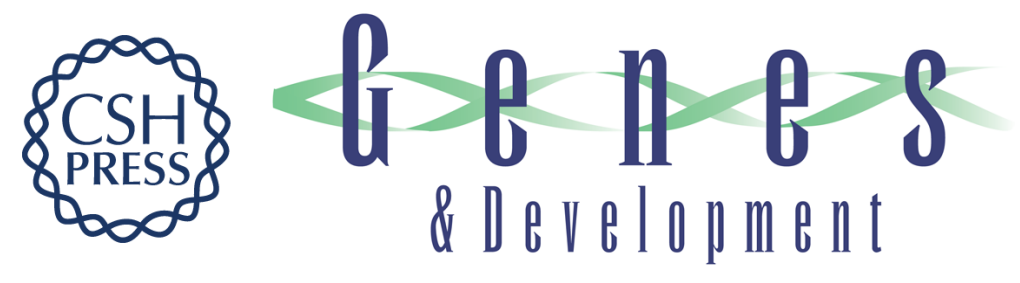

\section{Control of leaf vascular patterning by polar auxin transport}

Enrico Scarpella, Danielle Marcos, Jirí Friml, et al.

Genes Dev. 2006, 20:

Access the most recent version at doi:10.1101/gad.1402406

\footnotetext{
Supplemental http://genesdev.cshlp.org/content/suppl/2006/04/11/20.8.1015.DC1

Material

Related Content Polar auxin transport and patterning: grow with the flow Ben Scheres and Jian Xu

Genes Dev. UNKNOWN , 2006 20: 922-926

References This article cites 27 articles, 11 of which can be accessed free at: http://genesdev.cshlp.org/content/20/8/1015.full.html\#ref-list-1

Articles cited in:

http://genesdev.cshlp.org/content/20/8/1015.full.htmI\#related-urls

License

Email Alerting
Service $\begin{aligned} & \text { Receive free email alerts when new articles cite this article - sign up in the box at the top } \\ & \text { right corner of the article or click here. }\end{aligned}$
}

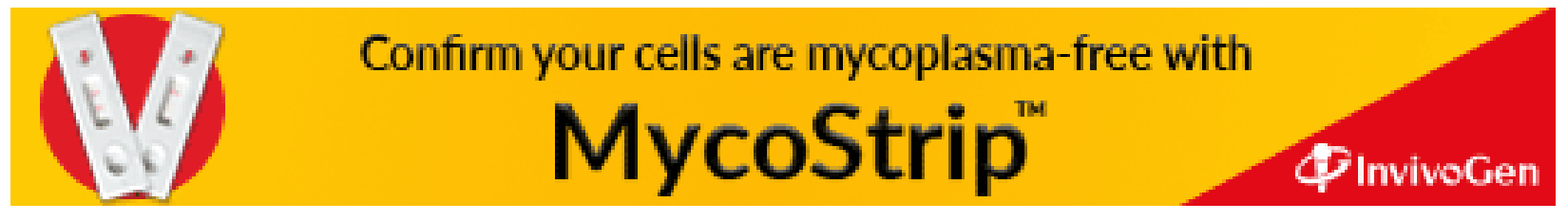

